\title{
Mechanism of phototaxis in marine zooplankton
}

\author{
Gáspár Jékely ${ }^{1} \dagger$, Julien Colombelli ${ }^{2}$, Harald Hausen ${ }^{3}$, Keren Guy $^{1}$, Ernst Stelzer $^{2}$, François Nédélec $^{2} \&$ Detlev Arendt $^{1}$
}

The simplest animal eyes are eyespots composed of two cells only: a photoreceptor and a shading pigment cell. They resemble Darwin's 'proto-eyes', considered to be the first eyes to appear in animal evolution ${ }^{1-4}$. Eyespots cannot form images but enable the animal to sense the direction of light. They are characteristic for the zooplankton larvae of marine invertebrates and are thought to mediate larval swimming towards the light. Phototaxis of invertebrate larvae contributes to the vertical migration of marine plankton ${ }^{5}$, which is thought to represent the biggest biomass transport on Earth ${ }^{6,7}$. Yet, despite its ecological and evolutionary importance, the mechanism by which eyespots regulate phototaxis is poorly understood. Here we show how simple eyespots in marine zooplankton mediate phototactic swimming, using the marine annelid Platynereis dumerilii as a model ${ }^{8}$. We find that the selective illumination of one eyespot changes the beating of adjacent cilia by direct cholinergic innervation resulting in locally reduced water flow. Computer simulations of larval swimming show that these local effects are sufficient to direct the helical swimming trajectories towards the light. The computer model also shows that axial rotation of the larval body is essential for phototaxis and that helical swimming increases the precision of navigation. These results provide, to our knowledge, the first mechanistic understanding of phototaxis in a marine zooplankton larva and show how simple eyespots regulate it. We propose that the underlying direct coupling of light sensing and ciliary locomotor control was a principal feature of the proto-eye and an important landmark in the evolution of animal eyes.

We assayed phototaxis of Platynereis larvae (Fig. 1a, b) in a dark chamber with $>750 \mathrm{~nm}$ transmission illumination. Larval swimming in a $1 \times 1 \times 0.2 \mathrm{~cm}$ cuvette in the dark chamber was recorded after providing a directional diffuse white light stimulus at one end of the chamber (Supplementary Fig. 1 and Supplementary Movie 1). We measured the efficiency of phototaxis by calculating the average displacement of the larvae towards the light (Fig. 1c). Positive phototaxis started at $25 \mathrm{~h}$ of development and lasted for two days. The spectral sensitivity was broad (365-545 nm) with two maxima at 410 and $490 \mathrm{~nm}$ (Fig. 1d), similar to that of phototactic larvae from other species ${ }^{9}$. Phototaxis could be triggered with intensities $>0.5 \mathrm{~mW} \mathrm{~cm}^{-2}$ using 500-nm monochromatic light. Phototactic larvae swim in a right-handed helix (that is, clockwise forward movement) of a diameter of $60-300 \mu \mathrm{m}$ while rotating around their anterior-posterior axis with one full turn every 0.6-0.9 s. During swimming the dorsal side of the larva is always directed towards the axis of the helix so that a $360^{\circ}$ axial rotation takes place in one pitch of the helix (Fig. 1e). This is a general phenomenon among protozoa and ciliated animal larvae $e^{10-14}$.

Platynereis larvae develop a pair of eyespots consisting of a pigment cell and a rhabdomeric photoreceptor cell ${ }^{8,15}$ (Fig. 1b, f). The pigment cup of the pigment cell is directly adjacent to the photosensitive rhabdom of the photoreceptor and shades it from one side (Fig. 1f-i). Both eyespots have wide, slightly dorsally and posteriorly tilted planes of view as determined by electron microscopy three-dimensional reconstructions of individual pigment vesicles and of the rhabdom outline (Fig. $1 \mathrm{~h}-\mathrm{j}$ ). The restricted view angle of the photoreceptors confers differential sensitivity to the direction of light that is thought to be the basis of phototaxis ${ }^{12,16}$. To test whether it is the eyespots and not the developing adult eyes or brain ciliary photoreceptors ${ }^{8,17}$ that mediate phototaxis, we performed bilateral ablations of eyespots using pulsed laser nanosurgery ${ }^{18,19}$ (Supple $>$ mentary Movie 2). This resulted in the complete loss of phototaxis ( 0 phototactic larvae out of 40 ablated ones) as opposed to ablation of two cells in the apical organ (27 phototactic larvae out of 32 ablated ones). In contrast, when we ablated only one eyespot most larvae were still able to swim towards the light (left eye ablated: 19 out of 24, right eye ablated: 13 out of 21 phototactic). This shows that eyespots work as independent units and that the comparison of light levels between the eyespots is not needed during phototaxis.

Platynereis larvae swim with the ciliary band consisting of two tiers of 12 large multiciliated cells (Figs 1a and 2a, b). Staining of all neurites with anti-acetylated-tubulin antibody showed axonal contact between the eyespot and the ring nerve that runs underneath the ciliary band (Fig. 2a). To trace in detail the axonal connections between the eyespots and the ciliated cells, we followed the photoreceptor cell through 70-nm electron microscopy sections. We found that the cell body of the photoreceptor extends a basal axon that joins the ring nerve and forms synapses on the multiciliated cells (Fig. 2b, c and Supplementary Fig. 2). Immunohistochemistry allowed further characterization of the photoreceptor axon. We identified the 7-to-9-amino-acid neuropeptide $\mathrm{x}(3,5)$-FVRIamide (in short, FVRI-amide) as a photoreceptor marker by co-staining the rhabdom microvilli using rhodaminephalloidine and the photoreceptor cell body using an antibody against FVRI-amide (Fig. 2d). FVRI-amide immunostainings also highlighted the photoreceptor axon along its entire length (Fig. 2e). In agreement with the electron microscopy data, we observed varicose thickenings adjacent to ciliated cells indicative of synaptic contact (Fig. 2e).

To identify the neurotransmitter of the photoreceptor we performed whole-mount in situ hybridization with candidate marker genes. Staining for Platynereis vesicular acetylcholine transporter ${ }^{20}$ (VAChT) identified a cholinergic neuron in the region of the eyespot (Fig. 2f). Double whole-mount in situ hybridization ${ }^{21}$ with VAChT and the photoreceptor-specific FVRI-amide neuropeptide precursor probe showed cellular-resolution co-expression ${ }^{22}$, demonstrating that the photoreceptor is cholinergic (Fig. $2 \mathrm{~g}$ ). We also found that an $\alpha 9 / \alpha 10$-subunit of nicotinic acetylcholine receptor is expressed in the ciliated cells (Fig. 2h). These results indicate that the eyespot photoreceptor works as a sensory-motor neuron that directly regulates phototactic steering by acetylcholine $(\mathrm{ACh})$. To test the involvement of ACh we performed phototaxis experiments in the presence of the ACh receptor antagonists mecamylamine and $\alpha$-bungarotoxin. The presence of both antagonists obliterated phototaxis without affecting the speed of swimming (Fig. 2i, Supplementary Fig. 3 and Supplementary Movie 3). 

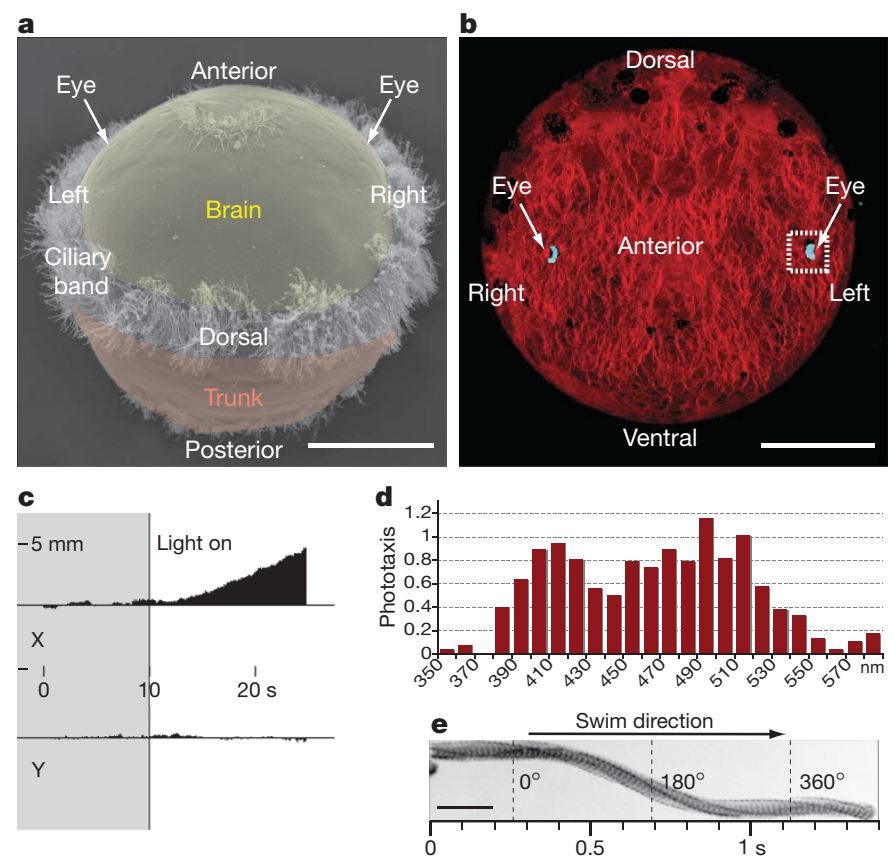

f

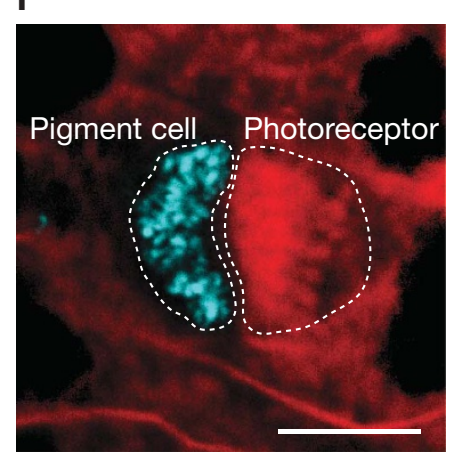

g

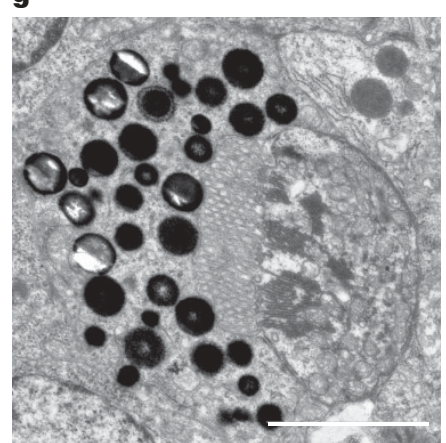

h

i
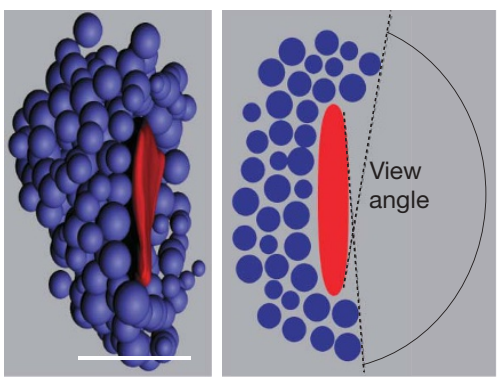

j

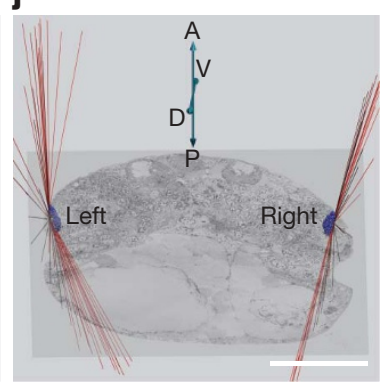

Figure 1 | Larval eyespots mediate phototaxis in Platynereis. a, Scanning electron micrograph of a 48-h larva. $b$, Anterior view showing pigment autofluorescence (cyan) with the membrane label bodipy564/570-propionic acid (red). Boxed region is enlarged in f. c, Quantification of average phototactic displacement with directional light after $10 \mathrm{~s}$. Positive phototaxis is indicated as positive $\mathrm{X}$ values $(n>200$ larvae). d, Action spectrum of phototaxis. Efficiency is measured as the slope of the displacement plots (compare to panel c; $n>150$ larvae). e, Superposition of video frames ( 60 frames per second (f.p.s.)) of a spiralling phototactic larva, illumination is from the right. The anterior end is forward, the anterior-posterior axis is parallel to the helical axis. The axial rotation is shown. f, Pigment (cyan) with bodipy564/570-propionic acid (red), the images is enlarged from $\mathbf{b}$. Cell outlines are shown. $g$, Electron microscopy section showing the pigment cup surrounding the photoreceptor rhabdom. h, Three-dimensional reconstruction of eyespot on the basis of electron microscopy sections. i, Schematic view showing how view angles were determined. $\mathbf{j}$, Reconstruction of the view angle of eyespots. The anterior-posterior (A-P) and dorsal-ventral (D-V) axes are indicated. Scale bars, $50 \mu \mathrm{m}(\mathbf{a}, \mathbf{b}, \mathbf{j}), 500 \mu \mathrm{m}(\mathbf{e}), 5 \mu \mathrm{m}(\mathbf{f})$ and $2 \mu \mathrm{m}(\mathbf{g}, \mathbf{h})$.
The direct cholinergic innervation of adjacent ciliated cells by the eyespot photoreceptor suggested a direct effect of light perception on ciliary beating. To test this we selectively illuminated one eyespot with a $500 \mathrm{~nm}$ monochromatic light beam of $10 \mu \mathrm{m}$ diameter (Supplementary Fig. 4) and recorded ciliary beating under $>650 \mathrm{~nm}$ transmission light. On illumination up to five ciliated cells on the side of the eyespot changed their beating pattern with a fast response time of $\sim 80 \mathrm{~ms}$ (Fig. 3a-c and Supplementary Movie 4 ). The response was inhibited by mecamylamine, consistent with a direct role for the cholinergic photoreceptor in triggering it (data not shown). The response involved a reduction in ciliary beat frequency (Fig. 3d) and an increase in the amplitude of the recovery stroke (Fig. 3e). No change was observed in either the beat pattern or the beat frequency in cells on the other side of the larva (Fig. 3d and data not shown). The ciliated cells that are closest to the eyespot, and can therefore be directly contacted by the photoreceptor axon, showed the strongest response (Fig. 3c, d). Under repeated stimulation with light/dark cycles ( $215 \mathrm{~ms}$ light, $215 \mathrm{~ms}$ dark) the response did not get attenuated (Fig. 3f), showing that the eyespot photoreceptor can quickly dark-adapt and is optimised to detect rapid intensity changes during axial rotation. To understand how the altered stroke pattern affects the forces exerted by cilia we visualized the water flow around immobilised larvae using $1 \mu \mathrm{m}$ microspheres (Figs $3 \mathrm{~g}$ and $4 \mathrm{a}$ ). We measured a decreased flow next to the affected cilia after selective illumination of one eyespot (Fig. 3h; dark speed ( \pm s.d.): $0.77 \pm 0.09$ $\mathrm{mm} \mathrm{s}^{-1}, n=36$ beads; light speed: $0.39 \pm 0.08 \mathrm{mms}^{-1}, n=29$ beads).

These results indicate that there is a functional difference between lateral 'steering' cilia that show a strong response, and dorsal-ventral 'non-steering' cilia that show no or a weak response. To test this difference experimentally we selectively removed all cilia from selected ciliated cell pairs using laser nanosurgery (Supplementary Movie 7) and performed phototactic turning assays. In this assay phototactic larvae had to turn $180^{\circ}$ after a sudden change in the direction of the phototactic light stimulus. When we ablated all cilia from cells 7 and 12, turning efficiency-as characterized by the diameter of the turning curves-was strongly reduced compared to non-ablated larvae or larvae with all cilia removed from cells 4 and 10 (Fig. 3i-k).

Next we wanted to understand the effect of such local change in the beating of the steering cilia on larval swimming and phototaxis. As a prerequisite we further characterized the flow generated by ciliary beating by video recordings. Using microspheres we observed a posteriorly directed flow tangent to the ciliary band (Fig. 4a) representing the main driving force of larval swimming. Recordings from apically oriented larvae showed a dorsal-ventral asymmetry in water flow: the six dorsal cell-pairs propelled microspheres slower than the ventral ones (Fig. 4b). Apically oriented larvae also showed a clockwise water flow around their circumference (data not shown). Taking these parameters into account we then mathematically modelled the swimming of Platynereis larvae, considering that they are spheres propelled in a viscous fluid ${ }^{23}$ by equatorial cilia that produce dorsoventrally asymmetric propulsion forces tangent to the sphere and torque that results in axial rotation (Fig. 4c). These parameters are sufficient to generate helical trajectories in a computer simulation of larval swimming (Fig. 4e, http://www.cytosim.org/platynereis). Such spiralling is usually the case for self-propelled objects, if the forces applied on the surrounding fluid are asymmetric and constant. In both phototaxis ${ }^{24,25}$ and chemotaxis ${ }^{26}$, the swimming forces have to be modulated to orient the trajectory towards the desired direction. To model this effect, we introduced local modulation of the ciliary beating forces as a function of the amount of light received by the eyespots. We numerically calculated the resulting trajectories and analysed them (see Supplementary Information for details). The model showed that a local change in ciliary propulsion force is sufficient to effectively steer larval swimming towards the light source (Fig. 4 d, e and Supplementary Movies 5 and 6). The trajectories were 

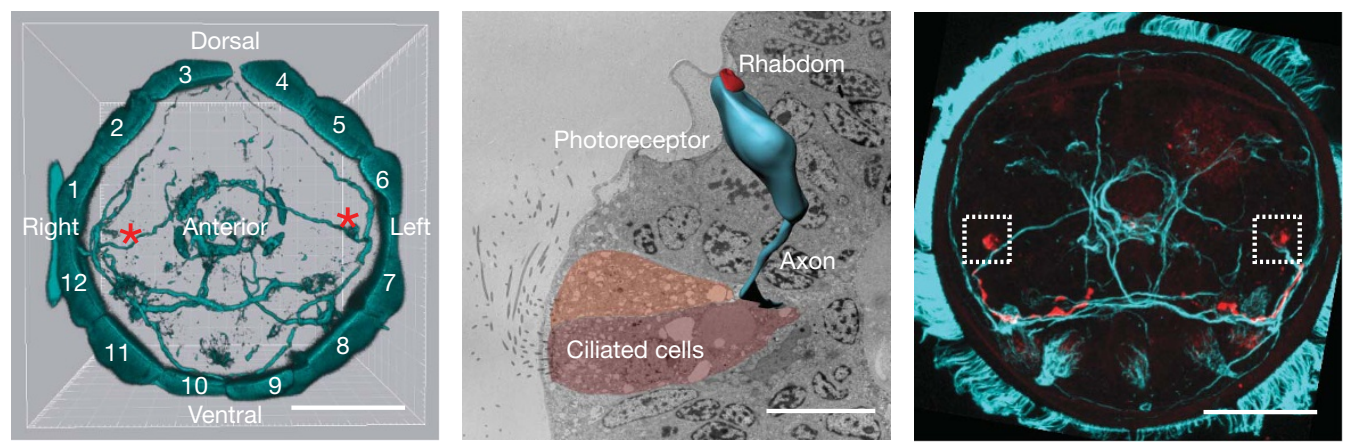

FVRI

acTub

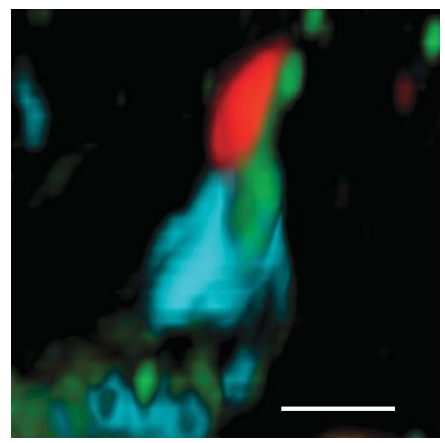

Phalloidin acTub FVRI

Figure $\mathbf{2}$ | The eyespot photoreceptor directly regulates phototaxis via ACh. a, Anterior view of larva immunostained for acetylated tubulin. Ciliated cells are numbered, the position of the eyespots is marked by red asterisks. b, Three-dimensional reconstruction of eyespot photoreceptor (cell body: cyan, rhabdom: red) and axonal contact to ciliated cells (two cells differentially coloured). c, Synaptic contact between the photoreceptor axon and a multiciliated cell. The axon is indicated by a white asterisk; the red arrowhead points to a synapse. d, Anti-FVRI-amide (cyan), anti-acetylated tubulin (acTub, green) and rhodamine-phalloidin (red) staining of the photoreceptor cell body, dendrite and rhabdom, respectively.
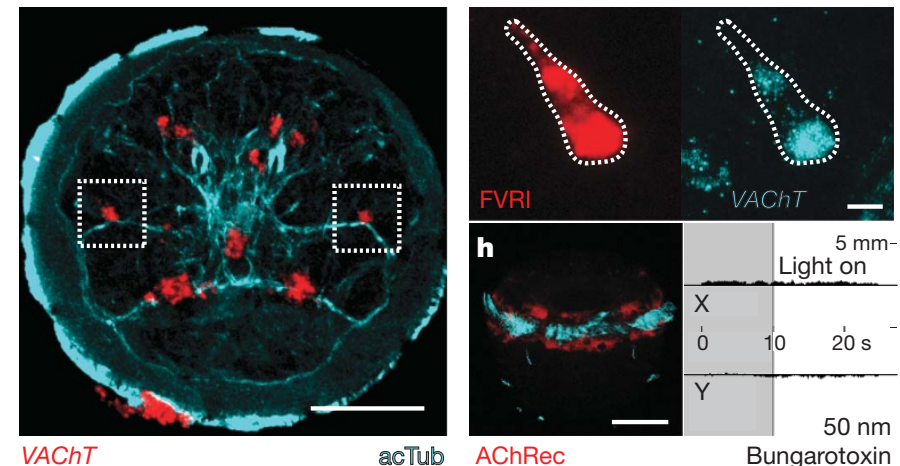

Bungarotoxin

e, Immunostaining for FVRI-amide (red) and acetylated tubulin (cyan).

f. Whole-mount in situ hybridization for VAChT (red), counterstained for acetylated tubulin (cyan). g, Double whole-mount in situ hybridization for FVRI-amide precursor (red) and VAChT (cyan), the outline of the cell is marked. $\mathbf{h}$, Whole-mount in situ hybridization for the $\alpha 9 / \alpha 10$-subunit of nicotinic ACh receptor (AChRec) counterstained for acetylated tubulin. i, Quantification of phototaxis in the presence of $50 \mathrm{nM} \alpha$-bungarotoxin $(n>200$ larvae; compare to Fig. 1c). d, $\mathbf{g}$ and $\mathbf{h}$ show lateral views, all other panels are anterior views. Scale bars, $50 \mu \mathrm{m}(\mathbf{a}, \mathbf{e}, \mathbf{f}, \mathbf{h}), 10 \mu \mathrm{m}(\mathbf{b}), 0.5 \mu \mathrm{m}$ (c) and $5 \mu \mathrm{m}(\mathbf{d}, \mathbf{g})$.

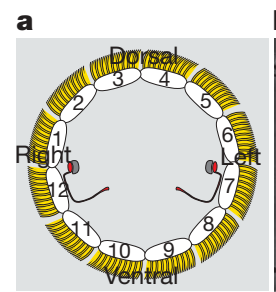

b
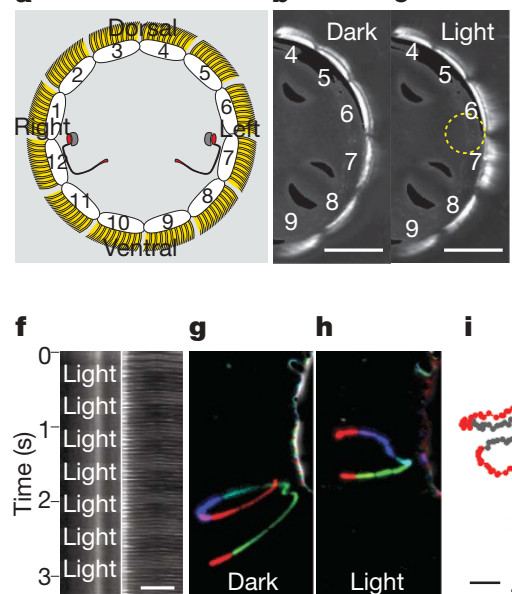

\begin{abstract}
g
\end{abstract}

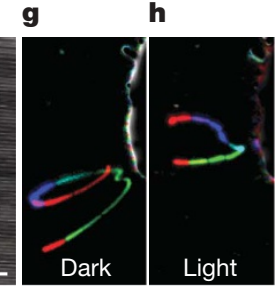

i

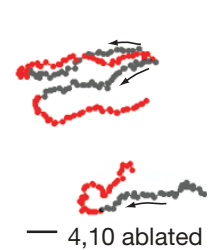

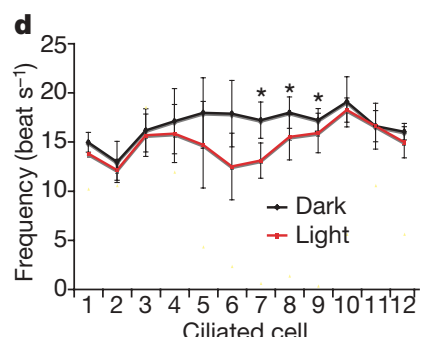

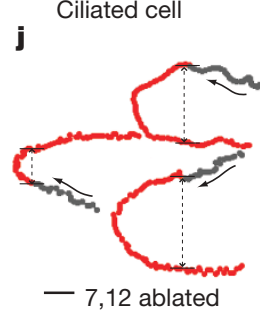

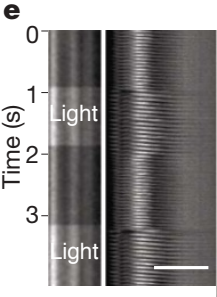

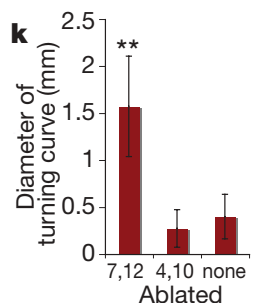

Figure 3 | Light regulation of ciliary beating. a, Schematic anterior view of a larva. b, c, Effect of local illumination on ciliary beating. Superposition of frames from a 60 f.p.s. movie taken in the dark (b) or when the left eyespot was illuminated locally (yellow dashed circle) (c). A change in beating pattern is apparent in cells 6 and 7 (c).d, Beat frequencies of cilia in the dark and under selective illumination of the left eyespot. Asterisks, $P<0.05$ (paired $t$-test), $n=3$ larvae. e, Kymograph from cilia from a laterally oriented larva during selective stimulation of the adjacent eyespot. f, Kymograph of cilia upon $215 \mathrm{~ms}$ light/dark selective stimulation of the adjacent eyespot. $\mathrm{g}, \mathrm{h}$, Superposition of frames from a 60 f.p.s. movie showing a microsphere propelled by ciliated cell pair 7 in the dark $(\mathbf{g})$ and after illumination of the left eyespot (h). i, j, Phototactic turning trajectories of larvae after $180^{\circ}$ reversal of directional light source with laser ablation of cells 4,10 (i) and 7,12 (j). k, Diameter of phototactic turning curves $(n=8$ larvae each). Double asterisk, $P<0.0001$ (unpaired $t$-test) between controls and 7,12 ablated larvae. Error bars, s.e.m. (d) and s.d. (k). Scale bars, $50 \mu \mathrm{m}$ (b, c), $10 \mu \mathrm{m}(\mathbf{e}, \mathbf{f}, \mathbf{g})$ and $100 \mu \mathrm{m}(\mathbf{i}, \mathbf{j})$. 

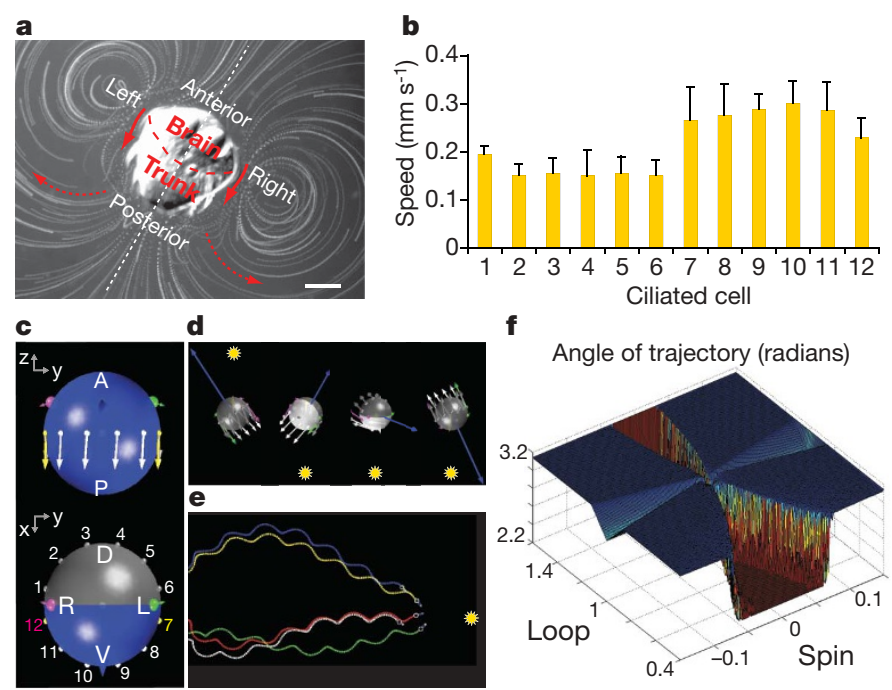

Figure 4 | Computer simulations of phototaxis. a, Flow around an immobilised laterally oriented larva. Superposition of video frames showing microspheres carried by cilia-generated flow. Red arrows show flow direction. The ciliary band is indicated with a dashed line. Scale bar, $50 \mu \mathrm{m}$. b, Quantification of the speed of microspheres propelled by each of the 12 ciliated cell pairs, error bars show s.d., $n>4$ beads. c, Simulated larvae are spherical and propelled by equatorial forces (arrows). The eyes detect light from the direction shown by small arrows and control the cilia 7 and 12. A, anterior; P, posterior; D, dorsal; V, ventral; R, right; L, left. d, Simulated reorientation to an infinite light source coming from the opposite direction (yellow dots). e, Simulated spiral phototactic trajectories of larvae swimming to a local light source (yellow spot on the right). f, Precision of phototaxis as a function of spin and loop. Colour tone indicates the angle (in radians) of the swimming trajectories to the light direction.

similar to the trajectories recorded for Platynereis larvae (Figs 1e and $4 \mathrm{e})$.

To gain further insights into the mechanism of phototaxis we used our computer model to test how varying the dorsal-ventral asymmetry in ciliary beating (loop factor), as well as the speed and direction of axial rotation (spin factor), would affect the efficiency of phototaxis. To scan the parameter space we ran 300,000 simulations and plotted the precision of phototactic steering as determined by the angle of the swimming trajectories to the light vector (Fig. 4f). This showed that when axial rotation is not present or is very slow (spin close to 0 ) phototactic orientation does not occur (Fig. 4f). These larvae only swim in a circle with a plane perpendicular to the light vector. Axial rotation restores the ability to steer in three-dimensions and is therefore essential for phototactic navigation. The plot also showed that straight-swimming larvae (that is, larvae with no dorsalventral asymmetry $($ loop $=1)$ but performing axial rotation $(\operatorname{spin} \neq 0))$, are imprecise and swim at an angle towards the light rather than directly towards it. This is due to an anterior blind zone in the view field of the larvae within which changes in light direction do not affect the orientation of larvae (compare with Fig. 1j). The spiralling resulting from the dorsal-ventral asymmetry (loop $\neq 1$ ) compensates for this and allows the larvae to scan the space in all directions. Our simulations thus reveal that the spiral swimming mode is necessary for precise three-dimensional navigation using simple direction sensing eyespots.

Spiral swimming is the rule among phototactic organisms ${ }^{10-14}$. It is generally observed in ciliated zooplankton larvae and also occurs in phototactic single-celled algae such as Chlamydomonas ${ }^{24,25}$. Although the swimming mechanics of Chlamydomonas is different, the strategy for phototaxis is similar (an eyespot with a restricted view field that rotates in a spiral and directly regulates the beating of cilia). In each case, eyespots and cilia-driven helical swimming are intimately linked functionally and co-adapted for efficient phototaxis. In Platynereis, this is reflected by the direct sensory-motor coupling between the eyespot photoreceptor and the locomotor ciliary cells. In sponge and cnidarian zooplankton larvae, directional light detection and ciliary locomotor output are combined in a single $\operatorname{cell}^{27,28}$. We therefore propose that such direct sensory-motor coupling between a light sensor cell and effector cilia mediating phototaxis represents an ancestral condition in the evolution of animal eyes.

\section{METHODS SUMMARY}

Behaviour. Platynereis larvae were obtained from an established breeding culture, following that previously described ${ }^{29}$, and were raised at $18^{\circ} \mathrm{C}$ in natural sea water. Behavioural experiments were performed in sea water using 34-52-h-old Platynereis larvae. Receptor antagonists were added to the sea water and larvae were incubated for $5 \mathrm{~min}$ before recordings. Water flow was highlighted using $1 \mu \mathrm{m}$ FluoSpheres (Invitrogen).

Laser ablations. Laser ablations of eyespots and cilia were performed on immobilised larvae using either the self-implemented EMBL laser nanosurgery ${ }^{18}$ Axiovert 200M microscope (Carl Zeiss), or a CellCut System from Olympus. Staining. Immunostainings and whole-mount in situ hybridization were performed using established protocols ${ }^{21,22}$ on precisely staged larvae. Reflection imaging of nitroblue tetrazolium (NBT)/5-bromo-4-chloro-3-indolyl phosphate (BCIP) stained whole-mount in situ hybridization samples was performed as described ${ }^{22}$.

Full Methods and any associated references are available in the online version of the paper at www.nature.com/nature.

\section{Received 11 September; accepted 27 October 2008.}

1. Darwin, C. On the Origin of Species by Means of Natural Selection: or the Preservation of Favoured Races in the Struggle for Life (John Murray, 1859).

2. Arendt, D.\& Wittbrodt, J. Reconstructing the eyes of Urbilateria. Phil. Trans. R. Soc. Lond. B 356, 1545-1563 (2001).

3. Gehring, W. J. \& Ikeo, K. Pax 6: mastering eye morphogenesis and eye evolution. Trends Genet. 15, 371-377 (1999).

4. Pichaud, F. \& Desplan, C. Pax genes and eye organogenesis. Curr. Opin. Genet. Dev. $12,430-432$ (2002).

5. Thorson, G. Light as an ecological factor in the dispersal and settlement of larvae of marine bottom invertebrates. Ophelia 1, 167-208 (1964).

6. Hays, G. C. A review of the adaptive significance and ecosystem consequences of zooplankton diel vertical migrations. Hydrobiologia 503, 163-170 (2003).

7. Angel, M. V. \& Pugh, P. R. Quantification of diel vertical migration by micronektonic taxa in the northeast Atlantic. Hydrobiologia 440, 161-179 (2000).

8. Arendt, D. et al. Development of pigment-cup eyes in the polychaete Platynereis dumerilii and evolutionary conservation of larval eyes in Bilateria. Development 129, 1143-1154 (2002).

9. Forward, R. B. Diel vertical migration: zooplankton photobiology and behaviour. Oceanogr. Mar. Biol. Annu. Rev. 26, 361-393 (1988).

10. Jennings, H. S. On the significance of the spiral swimming of organisms. Am. Nat. 35, 369-378 (1901).

11. Brandenburger, J. L., Woollacott, R. M. \& Eakin, R. M. Fine structure of eyespots in tornarian larvae (Phylum: Hemichordata). Z. Zellforsch. Mikrosk. Anat. 142, 89-102 (1973).

12. Marsden, J. R. Swimming in response to light by larvae of the tropical serpulid Spirobranchus giganteus. Mar. Biol. 83, 13-16 (1984).

13. Witman, G. B. Chlamydomonas phototaxis. Trends Cell Biol. 3, 403-408 (1993).

14. Maldonado, M. The ecology of the sponge larva. Can. J. Zool. 84, 175-194 (2006).

15. Rhode, B. Development and differentiation of the eye in Platynereis dumerilii (Annelida, Polychaeta). J. Morphol. 212, 71-85 (1992).

16. Lacalli, T. C. Structural correlates of photoresponse in trochophore larvae. Can. J. Zool. 66, 1004-1006 (1988).

17. Arendt, D. et al. Ciliary photoreceptors with a vertebrate-type opsin in an invertebrate brain. Science 306, 869-871 (2004).

18. Colombelli, J., Grill, S. W. \& Stelzer, E. H. K. Ultraviolet diffraction limited nanosurgery of live biological tissues. Rev. Sci. Instrum. 75, 472-478 (2004).

19. Colombelli, J., Reynaud, E. G. \& Stelzer, E. H. Investigating relaxation processes in cells and developing organisms: from cell ablation to cytoskeleton nanosurgery. Methods Cell Biol. 82, 267-291 (2007).

20. Denes, A. S. et al. Molecular architecture of annelid nerve cord supports common origin of nervous system centralization in Bilateria. Cell 129, 277-288 (2007).

21. Tessmar-Raible, K. et al. Fluorescent two-color whole mount in situ hybridization in Platynereis dumerilii (Polychaeta, Annelida), an emerging marine molecular model for evolution and development. Biotechniques 39, 460-464 (2005).

22. Jékely, G. \& Arendt, D. Cellular resolution expression profiling using confocal detection of NBT/BCIP precipitate by reflection microscopy. Biotechniques 42 , 751-755 (2007).

23. Denny, M. W. Air and Water: The Biology and Physics of Life's Media (Princeton Univ. Press, 1993).

24. Josef, K., Saranak, J. \& Foster, K. W. Ciliary behavior of a negatively phototactic Chlamydomonas reinhardtii. Cell Motil. Cytoskeleton 61, 97-111 (2005). 
25. Josef, K., Saranak, J. \& Foster, K. W. Linear systems analysis of the ciliary steering behavior associated with negative-phototaxis in Chlamydomonas reinhardtii. Cell Motil. Cytoskeleton 63, 758-777 (2006)

26. Friedrich, B. M. \& Jülicher, F. Chemotaxis of sperm cells. Proc. Natl Acad. Sci. USA 104, 13256-13261 (2007).

27. Leys, S. P. \& Degnan, B. M. Cytological basis of photoresponsive behavior in a sponge larva. Biol. Bull. 201, 323-338 (2001)

28. Nordström, K., Wallén, R., Seymour, J. \& Nilsson, D. A simple visual system without neurons in jellyfish larvae. Proc. Biol. Sci. 270, 2349-2354 (2003).

29. Dorresteijn, A. W. C. et al. Molecular specification of cell lines in the embryo of Platynereis (Annelida). Dev. Genes Evol. 202, 260-269 (1993).

Supplementary Information is linked to the online version of the paper at www.nature.com/nature.

Acknowledgements We thank K. Miura for advice on behavioural assays and digital image processing, K. Tessmar-Raible for identifying FVRI-amide in our expressed-sequence-tag collection, G. Plickert for advice on antibody production, D. Nilsson for advice on action spectra, and $\mathrm{H}$. Snyman for technical assistance. This work was supported by Center for Modelling and Simulation in the Biosciences and by grants from Deutsche Forschungsgemeinschaft (HA 4443/1-1 and DA Ar387/1-1). G.J. was supported by a FEBS Long Term Fellowship during the initial phase of the project

Author Contributions G.J. designed the experiments, characterized eyespots, performed behavioural experiments, coordinated the collaborations and wrote the paper. J.C. developed instrumentation for selective eye illumination and performed eyespot and cilia ablations. K.G. characterized FVRI expression. F.N. programmed and performed the simulations and wrote the paper. H.H. provided electron microscopy data and three-dimensional reconstructions. D.A. initiated the project, provided ideas and wrote the paper. All authors discussed the results and commented on the manuscript.

Author Information The sequences for the FVRI-amide neuropeptide precursor and the $\alpha 9 / \alpha 10$ subunit of nicotinic ACh receptor were deposited in the GenBank database with the accession numbers FJ358432 and FJ358433, respectively. Reprints and permissions information is available at www.nature.com/reprints. Correspondence and requests for materials should be addressed to G.J. (gaspar.jekely@tuebingen.mpg.de) or D.A. (arendt@embl.de). 


\section{METHODS}

Larva fixations. For immunohistochemistry and whole-mount in situ hybridization, larvae were fixed in 4\% paraformaldehyde in PBS $+0.1 \%$ Tween-20 and stored in methanol at $-20{ }^{\circ} \mathrm{C}$. For transmission electron microscopy, larvae were fixed in $2.5 \%$ glutaraldehyde in PBS for $1 \mathrm{~h}$ at $4{ }^{\circ} \mathrm{C}$ with traces of ruthenium red (Sigma), rinsed several times in PBS, and post-fixed with $1 \% \mathrm{OsO}_{4}$ in $\mathrm{PBS}$ for $20 \mathrm{~min}$ at $4{ }^{\circ} \mathrm{C}$. Larvae were dehydrated in a graded acetone series, transferred to propylene oxide and embedded in araldite for $48 \mathrm{~h}$ at $60^{\circ} \mathrm{C}$. Complete series of silver interfering sections were cut on a Leica EM UC6 microtome, placed on formvar (EMS)-coated cupper grids and stained with uranyl acetate and lead citrate. For scanning electron microscopy, larvae were fixed and dehydrated in the same manner, critically point dried in a Balzers CPD 030 (Balzers) and coated with gold in a Balzer Union SCD 040.

Antibody. An FVRI-amide peptide with an amino-terminal Cys was conjugated via the Cys residue to keyhole limpet hemocyanin, and used to immunise rabbits. The antisera were affinity purified on CFVRI-amide coupled to Sulfolink gel (Pierce).

Laser ablations. The Axiovert 200M microscope (Carl Zeiss) and the CellCut System from Olympus were both equipped with a subnanosecond pulsed laser at $355 \mathrm{~nm}$. Eyespot ablations were performed by localized intravital cavitation of the pigment cell induced with $30 \mathrm{~nJ}$ energy per pulse (with typically 10 laser pulses). If the larva was oriented upside-down and the laser beam had to focus through the scattering tissues energies of up to $1 \mu \mathrm{J}$ were used. Cilia removal was performed by cavitation in sea water at $5 \mu \mathrm{m}$ distance from the ciliated cell bodies. Energies in the range of 300-500 nJ were used (typically with 20 to 40 pulses along the targeted ciliary band cell), without apparent damage to the cell bodies. Simultaneous imaging of the ablated sample was only possible on the EMBL system with a Hamamatsu ORCA CCD camera.

Microscopy. Confocal images were taken using a Leica TCS SP2 confocal microscope with a $\times 40$ oil-immersion objective using the appropriate laser lines and a pinhole of airy unit 2. Transmission electron microscopy images were taken with a Phillips CM 120 Biotwin equipped with Ditabis Digital Imaging Plates. Threedimensional reconstructions were performed using Autodesk 3ds Max after manual alignment of image stacks. Scanning electron microscopy images were taken with a FEI Quanta 200 microscope. Recordings of phototactic larvae were done using a Leica MZ16 microscope equipped with a Sanyo VCB-3524P camera. Recordings of ciliary beating were done on immobilised larvae using a Zeiss Axiophot microscope equipped with a DMK 21BF04 camera (The Imaging Source). Larvae were immobilised between a slide and a coverslip spaced with adhesive tape.

Image processing. Stacks of confocal images or video recordings were processed using Image J 1.38J and Imaris 5.5.1. Contrast was adjusted equally across the entire image. Phototactic movies were analysed using a custom made ImageJ plugin.

Selective eyespot illumination. For selective eyespot illumination we used spectrally selectable monochromatic light (Till Photonics Polychrome II monochromator) coming through an optical guiding fibre of $1 \mathrm{~mm}$ diameter and coupled it into the back of the fluorescence port of a Zeiss Axiophot upright microscope. Through the use of a plano-convex lens (focal length $50 \mathrm{~mm}$, Melles Griot) and of an iris, placed $250 \mathrm{~mm}$ and $190 \mathrm{~mm}$ from the back lens of the port, respectively, we formed a disc of light onto the object plane of a $\times 100.3$ numerical aperture Zeiss objective lens. The diameter of the disc was $10 \mu \mathrm{m}$. The aperture of the iris did not exceed $500 \mu \mathrm{m}$ so that the numerical aperture of the beam at the sample location could be considered infinitely low (straight column of light). To allow axial displacement of the light disc, a manual X-Y mount holding a mirror was placed between the lens and the optical fibre. To perform switchable illumination we developed a software tool to control monochromator wavelength through a RS232 interface. 